\title{
Up Close: Center for Advanced Materials at Lawrence Berkeley Laboratory
}

\author{
John J. Gilman \\ Center for Advanced Materials \\ Lawrence Berkeley Laboratory
}

Editor's Note: This is the second article in a series focusing on the research capabilities and goals of interdisciplinary laboratories pursuing materials resiner in universities, industry, and government.

The boundaries between the present performance of materials and the requirements of device designers have for centuries been moving forward. The steps taken to draw these two together are sometimes large; more often they are small. As they occur, we find materials that are stronger, have larger magnetic moments, have higher electron mobilities, etc. Each time the property profile improves, understanding of the physical and chemical properties advances, and new engineering devices based on the improved profile are invented and developed.

The purpose of the Center for Advanced Materials (CAM) at the Lawrence Berkeley Laboratory ( $L B L$ ) is to enhance the interplay between advances in the property profiles of materials and advances in the chemical and physical understanding of them. For this purpose, the location of CAM can be described as ideal. The proximity of this national laboratory to the campus of the University of California at Berkeley provides an unusually rich in tellectual setting for the Center. It also provides unique opportunities for the University students and faculty who conduct materials-related research. Indeed, the arrangement should be a model for similar organizations, and it represents a solid method for strengthening materials science and technology throughout the nation.

National policy in critical materials has given the national laboratories-including LBL-strong direction and incentive to collaborate with ind ustry and the research universities. This incentive led to the es tablishment of CAM in order to build on the symbiosis between LBL and the University of California at Berkeley. It strives to extend this symbiosis by bringing industry into the ongoing educational process and by making its special facilities more readily available to industrial researchers.

Materials advances result from new compositions, new processes, and new architectures for materials in devices. All these areas have reached high levels of sophistication, and they are moving higher still. Large, complex tools for synthesis and analysis have, the refore, become necessary. For example, one materials research trend has been toward the control of structure at very small scales. This control can be achieved only by means of complex ma- chines for making the materials and analyzing the products. The infrastructure of a large laboratory facility is highly advantageous for such operations; in some cases it is a necessity. Within LBL, CAM is availed of special equipment such as powerful electron microscopes and particle accelerators, an infrastructure that includes highly developed computer and information systems, personnel with a broad variety of technical skills, and an exceptionally wellqualified cadre of graduate students and faculty.

CAM consists of six groups: (1) Surface Science and Catalysis-with emphasis on the synthesis and characterization of microporous solids (carbides and zeolites), surface compounds (that exist at bimetallic and metal-oxide interfaces) and organometallic compounds; (2) Light Alloys-as represented by aluminum-lithium alloys; (3) Instrumentation-new methods for investigating surface structure; (4) Polymershow they derive their structures and properties from processing factors; (5) Electronic Materials-especially gallium arsenide; and (6) Ceramic/Metal Interfacesadhesion and its enhancement.

A policy board of representatives from industry, academia, and government provides overall guidance to the six groups. Each group also has its own advisory board (mostly industry representatives) which links CAM groups and industry. Additionally, liaison with industry is provided through CAM's Industrial Participation Office. Dozens of visitors are hosted by this office each year, and visits are also made by CAM representatives to industrial sites. Thus, an active dialogue with industry is maintained. The involvement of industry through the advisory boards, and through other means that are being developed, helps focus on particular problem areas. Coordination of CAM personnel to focus on a limited number of areas is one of CAM's distinguishing features.

Two laboratory building s a re being built for the use of CAM. One is $30-40 \%$ complete, and ground is being broken for the other. Together they will provide space for about 285 people.

A good way to characterize an organization is through its current or anticipated output. For CAM, this includes: technical discoveries, data, and understanding contributed to the professional literature; individuals educated in materials science and technology; prototypes of new materials, processes, or devices; explicit interactions with ind ustry through topical workshops, joint projects, liaison, exchange programs, or transfer of prototype technology; and special equipment that can be operated by visiting users. These outputs can contribute significantly to the vigor of materials science and technology. Such vigor will generate many new opportunities for the producers, users, and students of materials.

The most exciting materials research of the recent past has focused on materials produced far from equilibrium and materials with controlled supramolecular structures. These themes will probably continue to be vigorously pursued in the future. There is some evidence that an important new theme will be complex molecular structures derived from in vitro biochemical reactions.

Many opportunities for new materials of the next decade have not even been conceived today, but exciting extensions of today's work will certainly be made. CAM expects to participate in some of these projects in addition to pursuing advances in the technologies of established materials.

Center for Advanced Materials

Lawrence Berkeley Laboratory

1 Cyclotron Road

Berkeley, CA 94720

Telephone: (415) 486-4755

Following his graduation from Columbia Liniversity, Dr. Gilman's career has combined teaching and ind ustrial RED. He founded the Materinls Research Center at what is now the Allied-Signal Corp. and has been on the facultie's of Brown University amil University of Illinois. $H^{\prime}$ is a member of the National Academy of Engineering.

UP CLOSE SERIES

Look for upcoming articles in this series on

Argonne National Laboratory and

Northwestern University's Materials Research Center. 